\title{
Developing the Mathematical Model of the Bipedal Walking Robot Executive Mechanism
}

\author{
Zhanibek Issabekov, Nakhypbek Aldiyarov \\ Satbayev University, Almaty \\ Kazakhstan
}

\begin{abstract}
The paper considers the accuracy of footstep control in the vicinity of the application object. The methodology of forming a simulation of the executive electro-hydraulic servomechanism is developed. The paper presents control algorithms in the dynamic walking mode. The issues of stabilization of the sensors installed in the soles are investigated. The description of the laboratory model and simulation of the main links of the exoskeleton, approximated to human parameters, allowing to insert the studied algorithms of motion of the executive mechanism into the program of automation of calculations of the links of motion are given. The authors for the first time simulated the bipedal walking robot using modern digital technologies, including the joint use of pneumatic electric drive. This paper proposes an automated control scheme for manipulators controlling immobilized human limbs. Considering the functions of the leg and the phases of movement, the structure scheme is chosen so that the same actuator performs several functions. This construction partially reduces the load on the person, because the drives of the various links due to their gravity can overturn a person. Using the kinematic structure of the model and the method of adaptive control of the manipulator, as well as replacing some movement parts with plastic material, the authors were successful in reducing the total weight by three times compared with foreign analogues, which is important for a sick person.
\end{abstract}

Keywords-Exoskeleton; manipulator; model; kinematics; dynamics

\section{INTRODUCTION}

In the 21th century, there arise more and more situations that demand from people with disabilities to perform a wide variety of works related to daily life. To help people, mobile robotics tools are being created which can be controlled via radio or cable. Relating to a person, being near the power source, he can control his functions by cable. However, as life shows, in order to expand human capabilities associated with his movement, control can be carried out by radio channel. Many scientists work for creation of the motion control algorithms of bipedal walking robots that move in various dynamic modes of movement: walking, running, jumping, etc., in other words, human motion simulation, which formed the basis for the study of using the theory for creation of mechanisms for energetically optimal regulation of human walking [1-2].

For these reasons, scientists develop exoskeletons that represent a technical device designed for physical relief of a person who performing different works thanks to load accommodation by the exoskeleton, provided that it repeats human biomechanics [1-3]. As one reason for the growing popularity of these devices, it is necessary to mention the areas of their possible application:

1) Military sphere.

2) Use by people with disabilities.

3) Elimination of consequences of various emergencies.

4) Use of heavy equipment in conditions of inapplicability.

5) Use in operations where it is possible to replace heavy equipment with human labor.

Exoskeletons are classified according to the following [3$5]$.

1) According to the power source and the drive operating principle: passive exoskeletons, active exoskeletons;

2) According to the drive: electric, pneumatic, hydraulic.

Exoskeletons of today are becoming a very powerful tool to assist soldiers and medical staff of specialized clinics with rehabilitation of patients who have suffered from limb diseases. It was found that the most of designed exoskeletons cannot be used for rehabilitation of patients with limited functions of the upper and lower limbs due to the large mass of the structure, dependence on external power sources and their significant cost. For designing the exoskeleton, it is necessary to solve a lot of technical problems, among which are the following: the problem of walking control; designing the executive mechanism using original design solutions; designing the system of interconnected drives; designing the power source with a high specific power; designing the system of sensitivity, orientation and navigation; designing the control system and designing its algorithms. As of today, there is a lot of versions of exoskeletons built using various drives (electric drive, hydraulic drive, pneumatic drive), but their practical use is very limited due to difficulties associated with an on-board energy source that can provide the exoskeleton with autonomy. However, this fact only serves as the progress accelerator and leads to the constant appearance of more and more new versions of exoskeletons [4-6]. The purpose of the research is the use of exoskeletons in medicine for the rehabilitation of patients with musculoskeletal direction. This article describes the mechanisms for controlling the feet and the center of mass of a human robot.

\section{RELEVANCE}

The works of many scientists considered the problems of foot elasticity when modeling human movements [5-6], [5-6], 
dynamic motion of the manipulator based on the closed kinematic chain with coordinate-parametric control, twoengined electric drives of coordinated rotation based on an asynchronous-valve cascade, dual-power machines [7-8], etc., developments of which facilitated the development of investigations in the field of the manipulator use for replacing the human legs. There are many examples in the world of using the developments of scientists to improve the situation of persons disabled from childhood and work, to return them to normal life, their participation in the Paralympic Games, etc. Fig. 1 shows the development of scientists of Moscow universities, active and passive exoskeletons, as well as the exoskeleton appearance on a man simulator.

It is of interest the ReWalk exoskeleton developed in Israel by ARGO Medical Technologies which allows people with paralysis of the lower half of the body (lower paraparesis) to stand on their feet and walk using sticks (Fig. 2).

The design of ReWalk exoskeleton is based on sensors that detect the body tilt ahead and transmit a signal to devices supporting the legs [4-7]. The power source is provided by a battery placed in a special backpack. It has 2 degrees of mobility. The structure operation is possible only for persons with preserved functions of the upper limbs.

A special feature of this layout diagram is installing 4 electric motors in the knee and hip joints of the exoskeleton, the ankle position is regulated by the springs that allow the foot to naturally stand on the ground (Fig. 3b).

A significant disadvantage of ReWalk exoskeleton is the complexity of ensuring the mechanism balance and hereto related safety problems of operator`s motion [9]. In most cases, these structural versions of exoskeletons are used as simulators for people undergoing rehabilitation.

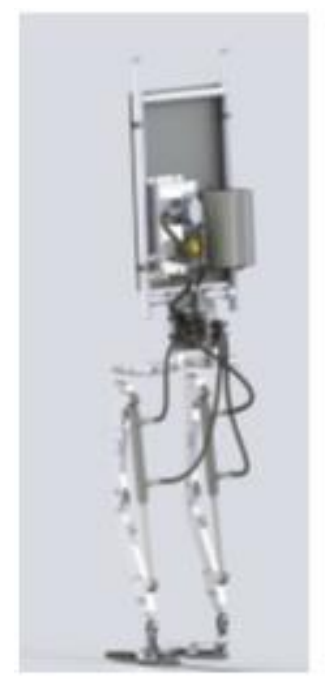

a)

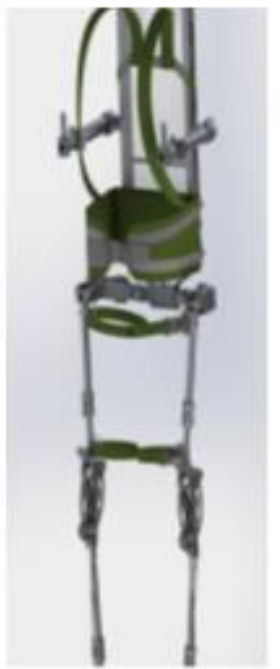

б)

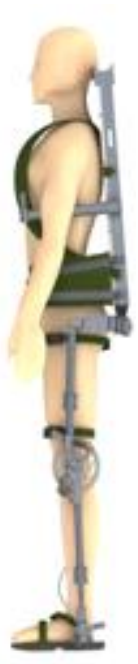

B) (a) active exoskeleton; (b) passive exoskeleton; (c) exoskeleton on a man simulator.

Fig. 1. D-model of Active and Passive Exoskeleton.

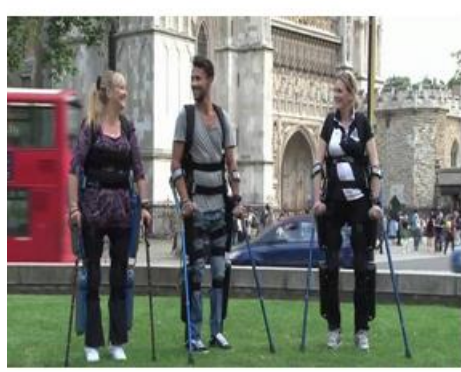

a)

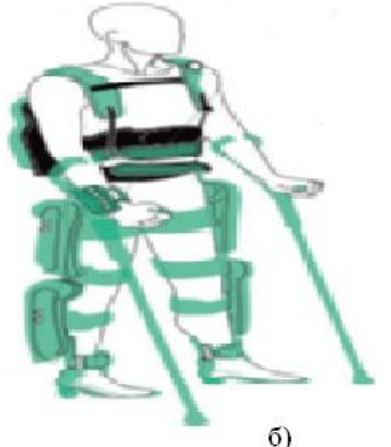

б)
Fig. 2. ReWalk Exoskeleton (a) and its Layout Diagram (b).

As far back as 1948, the Russian professor N. A. Bernstein drew a man with prosthetics copying the leg skeleton but with electric motors, which was the development of the Prosthetics Research Institute. It is interesting to note that just after the war ended, it was a very vital invention which, unfortunately, had no practical continuation in future. In the 60 `s, General Electric developed this idea but in a version of a full-fledged skeleton with hydraulic control. The same attempt was made by the Russian side in Russia (Leningrad, 1970) [5-6]. In Kazakhstan, such works are under development, so the study presented in this article is very relevant.

\section{Methodology}

A large number of investigations were devoted to the study of kinematics and dynamics of manipulation robots [7-9]. The manipulator executive mechanism has the treelike kinematical structure, a large number of mobility steps, does not properly secure to the fixed base, and during movement, it is connected with the supporting surface and ambient objects [10-11]. The authors for the first time develop a similar model with the use of modern digital technologies including the joint use of pneumatic-electric drive. This paper offers the automated control scheme for manipulators that control the immobilized human limbs. It is known that human manner of walking just as a robot determines by trajectories of the pelvis (main but dependent movement) and feet (auxilliary but forced movements). The main movement is carried out as a result of moving the legs [12-13]. The schematic structure of human musculoskeletal system is similar in many ways to the movement of bipedal walking robot which contains a system of solids and is in the form of closed kinematic chains with rotating and translating kinematic pairs connected to the body. Fig. 3 shows the model of human foot control in which relative movements of segments are carried out by drives. The leg mechanism has several degrees of mobility. Taking into account the leg functions and movement phases, schematic structure is chosen so that one and the same drive performs several functions. This construction partially reduces the load on the person, because the drives of the various links due to their gravity can overturn a person. 


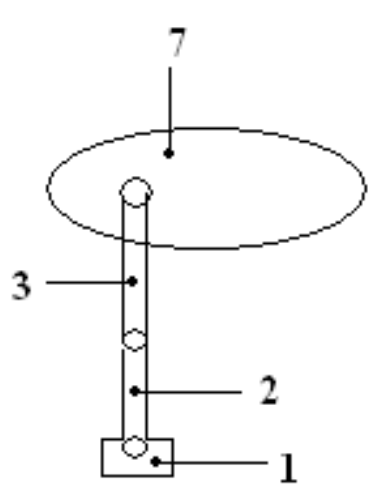

1- foot; 2-lower leg; 3-upper leg; 7- pelvic bone

Fig. 3. Human Leg Model.

Methods for describing the kinematic structure (KS) were developed for formation of mathematical models of the executive mechanism $[1,11]$.

To set the kinematic structure and write the kinematic expressions, the designations and indices given below were used. Kinematic connections of each segment are characterized by:

- number of one of the previous segment;

- numbers of one or more of subsequent segments;

- its sequential number for the previous segment.

Each segment is associated with as many coordinate systems as there are subsequent segments but not less than one. One of them is taken as the main one, which is assigned the number 1, the rest are auxiliary. All of them are assigned in accordance with the traditional Denavit-Hartenberg rules for robotics [1,9]. Vector values can be set in different coordinate systems (CS). Its upper left index indicates in which CS the given vector is specified. If this index is missing or null, then the vector is set in the base CS. If a nonzero number is specified, then the vector is specified in the main coordinate system of the segment, number of which is the specified number. If two numbers separated by commas are ${ }^{i, j} \bar{X}$, then the vector is specified in $j^{- \text {th }}$ the auxiliary coordinate system $i$. Let us denote by $\mathrm{L}=\{1,2,3 \ldots \mathrm{n}\}$ an unordered set, the elements of which are the numbers of the executive mechanism segments.

The following index functions are introduced: [11-13].

1) $\mathrm{f}(\mathrm{i})$ - segment number that is the parent segment for segment i.

2) $s(\mathrm{i}, \mathrm{k})-$-segment number that is $\mathrm{k}$-th son segment for segment i.

3) dg+ (i) - semidegree of segment $i$ that defines the number of son segments of $i$ segment. for

4) D(i) - cortege of numbers-links that are son segments segment $\left(\Gamma(\mathrm{i})=\left\{\left(\mathrm{s}(\mathrm{i}, 1), \mathrm{s}(\mathrm{i}, 2), \ldots, \mathrm{s}(\mathrm{i}, \mathrm{k}), \ldots, \mathrm{s}\left(\mathrm{i}, \mathrm{i}, \mathrm{dg}^{+}(\mathrm{i})\right)\right)\right\}\right)$;
5) ns(i) -defines what number as son segment has i segment for its father segment (sequence number of $i$ segment in the cortege. $\Gamma(\mathrm{f}(\mathrm{i}))$.

6) nan(i) - defines the number of ancestors of $i$ segment.

7) an $(i, j)$ - determines the number of the $j$-th from the root of the ancestor of the link i.

If $i$ segment is more than 0 , it is possible to write:

$n s(s(i, k))=k ; i=f(s(i, k)) ; \mathrm{k} \in\left\{1,2,3 \ldots d g^{+}(i)\right\}$

As it is for each segmenti(i $\in L$ ), we define the vector connecting the beginning of the father segment with the beginning of the son segment; the vector of the centre of mass (CM) position; the coefficient defining the type of articulation of main segment $i$ with father segment; the type of ancestor of segments - the rotary joint 1 ; the type of articulation of segments - the telescopic joint 0 .

This is how the exoskeleton robot segments are described and defined. However, if you transfer this system to a person, then you need to note the following: if the robot can turn and rotate according to the operator's instructions, then the person is limited in his motions. For example, the human foot (especially a sick person) cannot turn to $180^{\circ}$, as well as the knee and other parts of the lower limbs. Therefore, it is necessary to develop the motion algorithm limiting turns of feet from zero to $90^{\circ}$.

The method for describing kinematics of the bipedal walking robot executive mechanism was proposed by Denavit and Hartenberg with compatible coordinate systems all parameters of whose are presented in Fig. 4 [11-13].

The calculation system [4] is based on the use of homogeneous transformations matrices $(4 \times 4)$, which give unambiguous and clear rules for constructing a mathematical model of the robot's executive mechanism. At the same time, the number of parameters included into the matrix relative to the position of the successive segments of the executive mechanism is minimal. The matrix form Ai is identical both for rotational and translational joints.

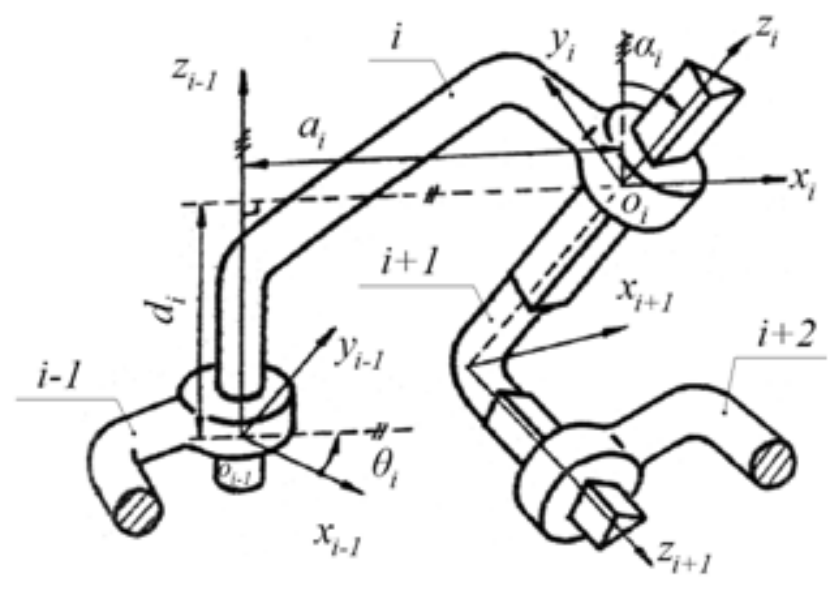

Fig. 4. Denavit-Hartenberg Compatible Coordinate Systems. 
The advantage of this method of constructing connected CS is that you can specify only four parameters that determine the relative position of two consecutive CS i-1 and i, and, consequently, the conversion matrix Ai. Two consecutive coordinate systems of segments, for example, $\mathrm{i}-1$ andi, can be always coincided using a rotation [14], two transfers and another rotation carried out by the $\mathrm{i}-1$ coordinate system in the following order:

- Rotate by an angle $\theta_{i}$ around the axis $z_{i-1}$ in the positive direction until the axes $\mathrm{x}_{\mathrm{i}-1}$ and $\mathrm{x}_{\mathrm{i}}$ become parallel and equally directed. If the joint is rotational, then the angle $\theta_{i}$ coincides with the generalized coordinate.

- Relocation over the distance $d_{i}$ along the axis $z_{i-1}$ until the axes $x_{i-1}$ and $x_{i}$ will coincide. If the joint is translational, then the $d_{i}$ coincides with the generalized coordinate.

- Relocation over the distance $\mathrm{a}_{\mathrm{i}}$ along the axis $\mathrm{x}_{\mathrm{i}}$ until the coordinate origin will coincide. Parameter $a_{i}$ is the constructive constant of the mechanism (depends on geometry of the structure).

- Rotation by the angle $\alpha_{\mathrm{i}} \alpha \mathrm{i}$ about the axis $\mathrm{x}_{\mathrm{i}}$ until all axes coincide.

Note that angles are positive if they are counted counterclockwise around the specified axes, and linear displacements are positive if they coincide with the positive directions of the corresponding axes.

\section{RESUlT AND DisCUSSION}

As a result of these movements the coordinate system $\mathrm{O}_{\mathrm{i}-1} \mathrm{x}_{\mathrm{i}-1} \mathrm{y}_{\mathrm{i}-1} \mathrm{z}_{\mathrm{i}-1}$ sequentially take up the possitions $\mathrm{O}_{\mathrm{i}-1}^{\prime} \mathrm{x}_{\mathrm{i}-1}^{\prime} \mathrm{y}_{\mathrm{i}-1}^{\prime} \mathrm{z}_{\mathrm{i}-1}^{\prime}, \mathrm{O}_{\mathrm{i}-1}^{\prime \prime} \mathrm{x}_{\mathrm{i}-1}^{\prime \prime} \mathrm{y}_{\mathrm{i}-1}^{\prime \prime} \mathrm{z}_{\mathrm{i}-1}^{\prime \prime}, \mathrm{O}_{\mathrm{i}-1}^{\prime \prime} \mathrm{x}_{\mathrm{i}-1}^{\prime \prime \prime} \mathrm{y}_{\mathrm{i}-1}^{\prime \prime \prime} \mathrm{z}_{\mathrm{i}-1}^{\prime \prime \prime}$ , and finally reaches the position $\mathrm{O}_{\mathrm{i}} \mathrm{x}_{\mathrm{i}} \mathrm{y}_{\mathrm{i}} \mathrm{z}_{\mathrm{i}}$. Moreover, each subsequent coordinate system is characteristed in the previous coordinate system by $4 \times 4$-coordinate transformation matrices sequentially.

From the four parameters $\theta_{\mathrm{i}}, \mathrm{d}_{\mathrm{i}}, \mathrm{a}_{\mathrm{i}}, \alpha_{\mathrm{i}}$ (Fig. 4), two parameters $\mathrm{a}_{\mathrm{i}}$ and $\alpha_{\mathrm{i}}$ are always constant and determined by the design of the robot executive mechanism. One of two other parameters $\left(\theta_{i}\right.$ ord $\left._{i}\right)$ is a variable parameter. For the rotational joint, the value $\theta_{\mathrm{i}}$ characterizes the angle of relative rotation of $\mathrm{i}-1$ and $\mathrm{i}$ segments, and the liner value $\mathrm{d}_{\mathrm{i}}$ is constant. Reverse, for the telescopic connection, $d_{i}$ is a variable. The variable of i-th joint $\left(\theta_{\mathrm{i}}\right.$ or $\left.\mathrm{d}_{\mathrm{i}}\right)$ is usually called the generalized coordinate of the robot executive mechanism [15-18].

Each limb has three degree of freedom and is derived by an engine with mechanism (transmission, gearbox, reduction unit). In the lower part of the limb there are three force sensors for measuring the leg force response.

Using the methods of describing the kinematic structure there were made the prerequisites for adaptive control of the executive mechanism for motion of any part of the human body. The authors considered the akinetic human limbs as a non-stationary object (NO). Some scientists propose to use the adaptive identification of NO with Markov parameters on the example of the bipedal walking robot. However, this NO is considered as a non-stationary dynamic multi-dimensional multi-loop controlled object with inputs $\mathrm{p}$ and outputs $\mathrm{q}$ (Fig. 5) [19-21].

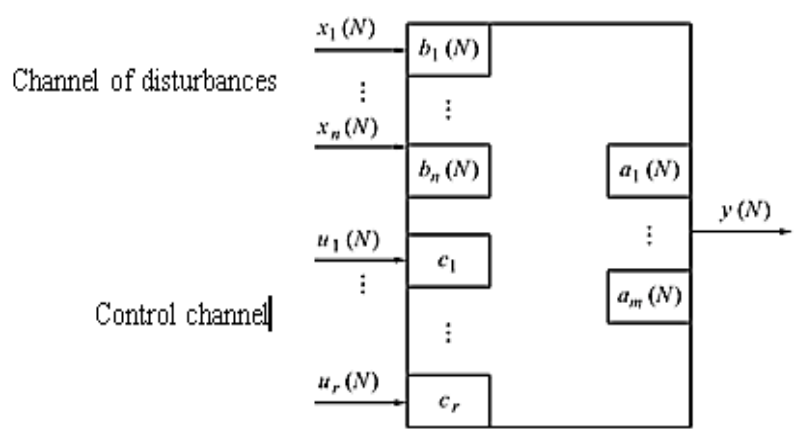

Fig. 5. Scheme of Non-stationary Dynamic Multi-dimensional Multi-loop Controlled Object.

There are two fundamentally different types of input channels at the object input. The perturbation channel is formed from $n$ observed inputs:

$\mathrm{X}^{\mathrm{T}}(\mathrm{N})=\left(\mathrm{x}_{1}(\mathrm{~N}), \ldots, \mathrm{x}_{\mathrm{n}}(\mathrm{N})\right)$, multi-loop controlled object $\mathrm{X} \in \mathrm{R}_{1}^{\mathrm{n}}$ where $\mathrm{R}_{1}^{\mathrm{n}}$ is a set of alloweble inputs with unobserved unknown parameters bj $(\mathrm{N}), \mathrm{j}=\overline{1, n}$.

The control channel is also observed: $\mathrm{UT}(\mathrm{N})=(\mathrm{u} 1(\mathrm{~N}), \ldots$, $\operatorname{ur}(\mathrm{N})), \mathrm{U} \in \mathrm{R}_{2}^{\mathrm{r}}$ where $\mathrm{R}_{2}^{\mathrm{r}}$ - a set of acceptable controls, but unlike the perturbation channel, all its parameters are known: $a_{1}(N) \ldots a_{m}(N)$, then $Y \in R_{3}^{q}$, where $R_{3}^{q}-a$ set of acceptable outputs $[13,22]$.

Using Denavit-Hartenberg method and the adaptive method of controlling the executive mechanism of human movement, the authors developed two variants of variables for the human exoskeleton changing $\mathrm{d}$ or $\mathrm{Q}$ parameter.

These models were put into Program of calculation and received (Table I).

The table shows the results of eight from 21 steps.

TABLE I. VALUES OF SIMULATED PARAMETERS OF EXOSKELETON SEGMENTS

\begin{tabular}{|l|c|l|l|l|l|l|}
\hline No. of step & $Q, \boldsymbol{r a d}$ & $\boldsymbol{d}, \mathrm{M}$ & $\boldsymbol{a}, \mathrm{M}$ & $\boldsymbol{\alpha}, \boldsymbol{r a d}$ & $\boldsymbol{f}(\boldsymbol{i})$ & $\boldsymbol{n s}(\boldsymbol{i})$ \\
\hline 1 & $-\pi / 2$ & 0 & 0 & $-\pi / 2$ & 0 & 1 \\
\hline 2 & $-\pi / 2$ & 0 & 0 & $-\pi / 2$ & 1 & 1 \\
\hline 3 & $-\pi / 2$ & 0 & 0 & $-\pi / 2$ & 2 & 1 \\
\hline 4 & $\pi / 2$ & 0 & 0 & $\pi / 2$ & 3 & 1 \\
\hline 5 & $\pi / 2$ & 0 & 0 & $\pi / 2$ & 4 & 1 \\
\hline 6 & 0 & $-0,39$ & 0 & $-\pi / 2$ & 5 & 1 \\
\hline 7 & $\pi / 2$ & $-0,1$ & 0,17 & $\pi / 2$ & 6 & 1 \\
\hline 8 & 0 & 0 & 0 & $-\pi / 2$ & 7 & 1 \\
\hline
\end{tabular}

It can be concluded from the table that angle $\mathrm{Q}$ changes from $-\frac{\pi}{2}, \frac{\pi}{2}, 0 \ldots d-0,39 ; d=-0,1 ; d=0$,etc. 
TABLE II. RESULTS OF ARBITRARY POINTS OBTAINED ON MATLAB

\begin{tabular}{|l|l|l|l|l|l|l|}
\hline No. of step & $\boldsymbol{Q}, \boldsymbol{r a d}$ & $\boldsymbol{d}, \mathrm{M}$ & $\boldsymbol{a}, \mathrm{M}$ & $\boldsymbol{\alpha}, \boldsymbol{r a d}$ & $\boldsymbol{f}(\boldsymbol{i})$ & $\boldsymbol{n s}(\boldsymbol{i})$ \\
\hline 6 & $-\pi / 2$ & 0 & 0,237 & $-\pi / 2$ & 5 & 1 \\
\hline 7 & 0 & 0,196 & 0,163 & $\pi / 2$ & 6 & 1 \\
\hline 8 & $\pi / 2$ & $-0,109$ & 0 & $-\pi / 2$ & 7 & 1 \\
\hline 9 & $\pi / 2$ & 0 & 0,487 & 0 & 8 & 1 \\
\hline 10 & 0 & 0 & 0,669 & $\pi / 2$ & 9 & 1 \\
\hline 11 & $\pi$ & 0,195 & $-0,097$ & $\pi / 2$ & 6 & 1 \\
\hline 12 & $\pi / 2$ & -0109 & 0 & $-\pi / 2$ & 11 & 1 \\
\hline 13 & $\pi / 2$ & 0 & 0,475 & 0 & 12 & 1 \\
\hline
\end{tabular}

It can be concluded from the Table II that angle $\mathrm{Q}$ changes from $-\frac{\pi}{2}, \frac{\pi}{2}, 0 \ldots d+0,196 ; d=-0,109 ; d=0$.

The authors developed and applied the Program to the model in Fig. 3.

\section{REFERENCES}

[1] Sh. Aszhan, "Development of an automated control system for the executive mechanism of a robot manipulator," Scientific and Technical Conference: Innovative Technology in Engineering. Almaty: ETU, pp. 176-179, April 2021.

[2] R.W. Jackson, C.L. Dembia, S.L. Delp and S.H. Collins, "Muscletendon mechanics explain unexpected effects of exoskeleton assistance on metabolic rate during walking", J. Exp. Biol., vol. 220, no. 11, pp. 20822095, 2017.

[3] N.T. Zhetenbaev, G.K. Balbaev, Zh. N. Isabekov and E.S. Nurgizat, "The future of robots with exoskeletonsin health care," Global science and innovations 2020: Central Asia, vol. 4, no. 3, pp. 26-32, 2020.

[4] Zh.N. Isabekov A.K. Kovalchuk and N.T. Zhetenbaev Exoskeletons of lower limitations: a brief explanation. Bulletin of KazATK, vol. 1, no. 108, pp. 78-84, 2019.

[5] J. Wu, J. Gao, R. Song, R. Li, Y. Li, L. Jiang, "The design and control of a 3D of lower limb rehabilitation robot," Mechatronics. vol. 33, pp.1322, 2016.

[6] A.K. Kovalchuk, "Designing drives of a medical robot actuator," Life Science Journal, pp. 337-340, 2014.

[7] D. Huamanchahua, Y. Tasa-Aquino, J. Figueroa-Bados, J. AlanjaVillanueva, A. Vargas-Martinez, R.A. Ramirez-Mendoza, "Mechatronic Exoskeletons for Lower-Limb Rehabilitation: An Innovative Review," 2021 IEEE International IOT, Electronics and Mechatronies Conference (IEMTRONICS), 2021. pp.1-8. doi: 10.1109/IEMTRONICS 52119.2021 .9422513$.
[8] G.V. Prado, R. Yli-Peltola, M.B.C. Sanchez, "Design and analysis of a lower limb exoskeleton for rehabilitation," Interdisciplinary Applications of kinematics, 2019, pp. 103-114.

[9] Zh.N. Isabekov, "Mathematical model of kinematics and dynamics of arbuscular executive machinery of proactive exoskeleton," Polytechnic youth magazine, Bauman MSTU, vol. 4, no. 4, pp. 5-10, 2016.

[10] A.K. Kovalchuk, "Modified Denavit-Hartenberg Coordinate System for Robot Actuating Mechanismus with Tree-like Kinamatic Structure," Science and Education of the Bauman MSTU, vol. 11, pp. 12-30, 2015.

[11] Zh.N. Issabekov, K.A. Moroz, M.F. Kerimzhanova, "Study of the dynamics of the exoskeleton actuating unit," Bulletin of BSTU named after V.G. Shukhov, no. 11. pp. 99-105, 2021. doi: 10.34031/20717318-2021-6-11-00-00.

[12] D.A. Elias, D. Cerna, C. Chicoma and R. Mio, "Characteristics of a lower limb exoskeleton for gait and stair climbing therapies," Interdisciplinary Applications of Kinematics, Springer, pp. 81-92, 2019.

[13] Z.N. Issabekov, I.K. Tsybrii, K.A. Moroz, "Organization of walking of the lower-extremity exoskeleton using the control of the supporting foot. Advanced Engineering Research," Series Machine Building and Machine science. 2021;21(3):247-252.

[14] Sh. Aszhan, "Requirements for the accuracy of control of the lower extremities of the exoskeleton near the object," International Scientific and Practical Conference: Modern Kazakhstan: reforms of education and science. Almaty: ETU, October 2021, pp. 248-251.

[15] A.K. Kovalchuk, "Method of mathematical description of the kinematics and dynamics of tree-like actuators of walking robots," Natural and Technical Science, vol. 5, no. 73, pp.87-90, 2014.

[16] A.K. Tanyildizi, O. Yakut, B. Tasar, "Mathematical modeling and control of lower extremity exoskeleton," Biomedical Research, vol. 29, no. 9, pp. 1947-1952, 2018.

[17] E.K. Lavrovsky, E.V. Pis'mennaya, P.A. Komarov, "On the problem of organizing the walking of the exoskeleton of the lower extremities using control deficit," Russian Journal of the Biomekhanics, no. 2, pp. 158176, 2015.

[18] T.M. Mukhidinov and K.S. Sholanov, "The movement of the biped walking robots," European research, vol. 1, no. 2, pp. 9-14, 2015.

[19] A.Zh. Toygozhinova, Research and development of automated deployment of air ozonisation, PhD thesis, Almaty, pp. 98-109, 2017.

[20] Song S., Collins S.H. Optimizing Exoskeleton Assistance for Faster Self-Selected Walking. IEEE Transactions on neural Systems and Rehabilitation Engineering, vol. 29, pp. 786-795, 2021. doi: 10.1109/TNSRE.2021.3074154.

[21] N.T. Isembergenov, K.N. Taissariyeva, U.O. Seidalieva, V.V. Danilchenko, "Microprocessor control system for solar power station," News of National Academy of sciences of the Republic of Kazakhstan. Series of geology and technical sciences, vol.1, no. 433, pp. 107-111, 2019. https://doi.org/1032014/2019.2518-170X.13.

[22] E.K. Lavrovsky, E.V. Pis'mennaya, "On the lower limb exoskeleton regular locomotion under input control deficit," Russian Journal of the Biomekhanics, vol. 18, no. 2, pp. 208-225, 2014. 